Annales Academiæ Scientiarum Fennicæ

Series A. I. Mathematica

Volumen 1, 1975, 125-142

\title{
EIN ABSTRAKTES CAUCHYSCHES PROBLEM
}

\author{
VEIKKO T. PURMONEN
}

\section{Einleitung}

In der vorliegenden Arbeit untersuchen wir die abstrakte Gleichung

$$
u^{\prime \prime}(t)+A u(t)+B u(t)=f(t)
$$

im Bochnerraum $L^{2}(I ; H)$ mit gewissen Anfangsbedingungen. Dabei benutzen wir sowohl für die Bochnerräume als auch im anwendenden Teil der Arbeit für die Lebesgue- und Sobolevräume den von P. Werner und J. Drehmann entwickelten distributionentheoretischen Aufbau der Theorie dieser Räume: Diese Räume werden dabei unter Benutzung ihrer funktionalanalytischen Eigenschaften ohne den Begriff des Lebesgueschen Integrals definiert; man siehe etwa [3], [9]. Eine allgemeine mathematische Theorie für die der zu betrachtenden Aufgabe verwandten Probleme ist von Ol'ga A. Ladyženskaja, M. I. Višik, C. H. Wilcox und anderen entwickelt worden (wegen dieser Theorie sowie des historischen und physikalischen Hintergrunds sei auf [5], [8], [10] verwiesen).

In $\S 1$ wird der von einer sesquilinearen Form definierte unbeschränkte Operator bezüglich eines Hilbertraumpaars betrachtet. Dann in $\S \S 2$ bis 5 formulieren wir ein abstraktes Cauchysches Problem (Aufgabe $(\boldsymbol{H})$ ) und beweisen, daß sie korrekt gestellt ist. Die Technik im Existenzbeweis in $\S 4$ ist ähnlich der z. B. in [3] für eine Rand- und Anfangswertaufgabe angewandten (man vergleiche auch [5]). Modifikationen verursacht jedoch die Tatsache, daß wir für unser abstraktes Problem nicht die Theorie der Dirichletschen Randwertaufgaben benutzen können. Zum Schluß der Arbeit wenden wir in $\S 6$ Aufgabe $(\mathcal{H})$ auf eine konkrete, für eine hyperbolische lineare partielle Differentialgleichung gestellte Cauchysche Anfangswertaufgabe mit nichthomogenen Randbedingungen (Aufgabe $(\boldsymbol{H} b d)$ ) an. Wir zeigen auch, daß die in [1], [2], [3] betrachtete Aufgabe mit homogenen Randbedingungen als ein Spezialfall in Aufgabe $(\mathcal{H} b d)$ enthalten ist.

Im Literaturverzeichnis sind nur die explizit referierten Schriften angegeben. 


\section{Der von einer sesquilinearen Form definierte (unbeschränkte) 0perator}

1.0. Man bezeichnet mit $N$ bzw. $N_{+}$die Menge der nichtnegativen bzw. positiven ganzen Zahlen, mit $\boldsymbol{R}^{n}$ bzw. $\boldsymbol{C}^{n}$ den $n$-dimensionalen $\left(n \in \boldsymbol{N}_{+}\right)$ reellen bzw. komplexen euklidischen Raum, $R=\boldsymbol{R}^{1}, \quad C=\boldsymbol{C}^{1}$, und mit $|\cdot|$ die entsprechende Norm. Für $z=x+i y \in C$ sei $\operatorname{Re} z:=x$ und $\bar{z}:=x-i y$.

Für zwei lineare topologische Räume $E$ und $F$ bedeute $\mathcal{L}\left(E ; F^{\prime}\right)$ die Menge der stetigen linearen Abbildungen $E \rightarrow F$.

1.1. Es seien $H$ und $V$ zwei separable (komplexe) Hilberträume versehen entsprechend mit den Skalarprodukten $(\cdot, \cdot)$ und $(\cdot, \cdot)_{V}$ sowie mit den zugehörigen Normen $\|\cdot\|$ und $\|\cdot\|_{V}$. Wir sagen, daß die Räume $H$ und $V$ ein Raumpaar $H, V$ bilden, wenn gilt:

$$
\begin{gathered}
V \subset H \text { algebraisch und topologisch, } \\
V \text { ist dicht in } H .
\end{gathered}
$$

1.2. Wir betrachten eine sesquilineare Form

$$
a(\cdot, \cdot): V \times V \rightarrow C
$$

und bezeichnen die entsprechende quadratische Form mit $a(\cdot)$,

Es gilt

$$
a(v):=a(v, v), \quad v \in V .
$$

Satz 1.1. (Lax-Milgram). Die Form $a(\cdot, \cdot)$ sei stetig und koerzitiv in $V, d$.h. es gibt positive Konstanten $K_{a}$ und $k_{a}$ mit

$$
|a(u, v)| \leqq K_{a}\|u\|_{V}\|v\|_{V}, \quad u, v \in V,
$$

und

$$
|a(v)| \geqq k_{a}\|v\|_{V}^{2}, \quad v \in V .
$$

Dann existieren für jedes $l \in \mathcal{L}(V ; C)$ eindeutige Elemente $v \in V$ und $w \in V$ mit

$$
l u=a(u, v)=\overline{a(w, u)}, \quad u \in V .
$$

1.3. Mit Hilfe des Satzes von Fréchet und Riesz verknüpft man mit der stetigen Form $a(\cdot, \cdot)$ einen Operator $A_{V} \in \mathcal{L}(V ; V)$, der der Relation

$$
a(u, v)=\left(A_{V} u, v\right)_{V}=\left(u, A_{V}^{*} v\right)_{V}, \quad u, v \in V,
$$

genügt, wobei $A_{V}^{*}$ der zu $A_{V}$ in $V$ adjungierte Operator ist. Leicht einzusehen ist 
Lemma 1.2. Unter den Voraussetzungen von Satz 1.1 ist der Operator $A_{V}\left(b z w . A_{V}^{*}\right)$ ein Isomorphismus von $V$ auf sich selbst.

1.4. Man bezeichne mit $U$ die Menge derjenigen $u \in V$, für die die antilineare Abbildung

$$
v 1 \rightarrow a(u, v): V \rightarrow C
$$

stetig bezüglich der $H$-Topologie ist. Für die stetige Erweiterung dieser Abbildung auf $H$ gibt es, wieder nach dem Satz von Fréchet und Riesz, ein $w \in H$ mit

$$
a(u, v)=(w, v), \quad v \in V .
$$

Durch $A u:=w, \quad D(A):=U$ erhalten wir einen linearen Operator $A: D(A) \rightarrow H$, der die Gleichung

$$
a(u, v)=(A u, v), \quad u \in D(A), \quad v \in V,
$$

erfüllt. Den Operator $A$ nennen wir den von $a(\cdot, \cdot)$ definierten (im allgemeinen unbeschränkten) Operator bezüglich des Raumpaars $H, V$. Entsprechend findet man einen Operator $A^{*}: D\left(A^{*}\right) \rightarrow H$ mit

$$
a(u, v)=\left(u, A^{*} v\right), \quad u \in V, v \in D\left(A^{*}\right),
$$

und demzu gilt

$$
(A u, v)=\left(u, A^{*} v\right), \quad u \in D(A), \quad v \in D\left(A^{*}\right) .
$$

Wir geben hier einen einfachen Beweis für

Satz 1.3. Es sei $H, V$ ein Raumpaar, und die Form $a(\cdot, \cdot)$ erfülle die Annahmen von Satz 1.1. Dann ist der Operator $A: D(A) \rightarrow H$ $\left(A^{*}: D\left(A^{*}\right) \rightarrow H\right)$ bijektiv.

Beweis. A. Der Operator $A$ ist injektiv; ist nämlich $u \in D(A)$ mit $A u=0$, so folgt

$$
k_{a}\|u\|_{V}^{2} \leqq|a(u)|=|(A u, u)|=0,
$$

d. h. $u=0$.

B. Die Epijektivität von $A$ ergibt sich daraus, daß die Gleichung

$$
a(u, v)=(h, v), \quad v \in V,
$$

lösbar in $V$ für jedes $h \in H$ ist. In der Tat, wegen der Stetigkeit der linearen Abbildung

$$
v \mapsto(v, h)=\overline{(h, v)}: V \rightarrow C
$$

gibt es nach Satz 1.1 ein $u \in V$ mit 


$$
\overline{(h, v)}=\overline{a(u, v)}, \quad v \in V,
$$

d. h.

$$
a(u, v)=(h, v), \quad v \in V .
$$

Infolge von Lemma 1.2 und Satz 1.3 hat man

Folgerung 1.4. Unter den Voraussetzungen von Satz 1.3 ist der Operator $A^{-1}: H \rightarrow D(A)$ stetig, und $D(A)$ ist dicht sowohl in $H$ als in $V$.

\section{Aufgabe (H)}

2.0. Zuerst führen wir einige Bezeichnungen ein. Für einen Banachraum $X$ und für ein Intervall $J \subset R$ bezeichne $C(J ; X)=C^{0}(J ; X)$ bzw. $C^{k}(J ; X), \quad k \in N_{+}$oder $k=\infty$, die Menge der (stark) stetigen bzw. $k$-mal stetig differenzierbaren Funktionen $J \rightarrow X$. Man schreibe $u^{(k)}=$ $(d / d t)^{k} u, \quad k \in N_{+}$, und kurz auch $u^{\prime}=u^{(1)}$ usw.. Es sei noch $C_{0}^{\infty}(J ; X)$ die Menge derjenigen $u \in C^{\infty}(J ; X)$, deren Träger Supp $u$, d. i. die Abschließung der Menge $\{t \in J \mid u(t) \neq 0\}$, kompakt und in $J$ enthalten ist.

Nun sei $H$ wie in 1.1 gegeben, und $I(\subset R)$ sei ein offenes Intervall $(0, T), \quad 0<T<\infty$. Mit der Topologie von L. Schwartz versehen wird $C_{0}^{\infty}(I ; H)$ der Testfunktionenraum $\mathcal{D}(I ; H)$. Den Raum der Distributionen $I \rightarrow H$ definieren wir als den topologischen Dualraum $D^{\prime}(I ; H)=$ $\mathcal{L}(\mathcal{D}(I ; H), C)$ von $\mathcal{D}(I ; H)$. Es sei bemerkt, daß im betrachteten Fall diese Definition der üblichen Definition $\mathcal{D}^{\prime}(I ; H)=\mathcal{L}(\mathcal{D}(I) ; H)$ äquivalent ist (dafür ist tatsächlich hinreichend, daß $H$ ein reflexiver Banachraum ist).

Im Raum $C(\bar{I} ; H)(\tilde{I}=[0, T])$ führen wir ein Skalarprodukt $((\cdot, \cdot))_{c}$ durch das Riemannsche Integral

$$
((u, v))_{c}:=\int_{0}^{T}(u(t), v(t)) d t, \quad u, v \in C(\bar{I} ; H),
$$

ein und bezeichnen die entsprechende Norm mit $\||\cdot|\|_{c}$. Den Bochnerraum $L^{2}(I ; H)$ definiert man jetzt durch $L^{2}(I ; H):=\mathcal{L}\left(C_{0}^{\infty}(I ; H) ; C\right)$, wobei $C_{0}^{\infty}(I ; H)$ mit dem Skalarprodukt $((\cdot, \cdot))_{c}$ versehen ist. Dann ist $L^{2}(I ; H)$ ein Banachraum, der durch eine isometrische Einbettung die Menge $C(I ; H)$ enthält (das entsprechende Element in $L^{2}(I ; H)$ des Elements $u \in C(\bar{I} ; H)$ wird auch mit $u$ bezeichnet). Die Norm $\||\cdot|\|$ von $L^{2}(I ; H)$ ist die Erweiterung von $\||\cdot|\|_{c}$ und wird tatsächlich von dem durch die Erweiterung von $((\cdot, \cdot))_{c}$ erklärten Skalarprodukt $((\cdot, \cdot))$ erzeugt; deswegen ist $L^{2}(I ; H)$ ein Hilbertraum. 
Nun gilt $L^{2}(I ; H) \subset \mathcal{D}^{\prime}(I ; H)$, und demzufolge kann man für $u \in L^{2}(I ; H)$ und für jedes $k \in N_{+}$die Ableitung $D_{t}^{k} u$ der Ordnung $k$ durch

$$
\begin{aligned}
\left\langle\left\langle D_{t}^{k} u, \varphi\right\rangle\right\rangle: & =(-1)^{k}\left\langle\left\langle u, \varphi^{(k)}\right\rangle\right\rangle \\
& =(-1)^{k}\left(\left(u, \frac{\varphi^{(k)}}{}\right)\right), \quad \varphi \in \mathcal{D}(I ; H),
\end{aligned}
$$

erklären, wobei $\langle\langle\cdot, \cdot\rangle\rangle$ das bilineare Dualprodukt zwischen $\mathcal{D}(I ; H)$ und $\mathcal{D}^{\prime}(I ; H)$ bedeutet.

2.1. Wir benutzen die Bezeichnungen von $\S 1$ und nehmen an, daß die Voraussetzungen von Satz 1.3 erfüllt sind und daß die Form $a(\cdot, \cdot)$ hermitesch ist, d. h.

$$
a(u, v)=\overline{a(v, u)}, \quad u, v \in V .
$$

Es sei weiter ein Operator $B \in \mathcal{L}(V ; H)$ gegeben; es besteht mit einer Konstanten $K_{b}>0$ die Ungleichung

$$
\|B v\| \leqq K_{b}\|v\|_{V}, \quad v \in V .
$$

2.2. Nun stellen wir die folgende abstrakte Cauchysche Aufgabe:

Aufgabe $(\mathcal{H})$. Es seien $g_{0} \in D(A), g_{1} \in V$ und $f \in C^{1}(\bar{I} ; H)$ gegeben. Gesucht ist eine Funktion $u$ mit den Eigenschaften:

$$
\begin{aligned}
& u \in C^{2}(\vec{I} ; H) \cap C^{1}(\bar{I} ; V), \\
& u(t) \in D(A), \quad t \in \tilde{I}, \\
& u^{\prime \prime}(t)+A u(t)+B u(t)=f(t), \quad t \in \bar{I}, \\
& u(0)=g_{0}, \quad u^{\prime}(0)=g_{1} .
\end{aligned}
$$

Jedes solche u nennen wir eine Lösung der Aufgabe.

Wir werden in $\S \S 3$ bis 5 zeigen, daß Aufgabe $(\mathcal{H})$ in dem in $\S 5$ definierten Sinne korrekt gestellt ist (Satz 5.1).

Bemerkung. Die Annahme $f \in C^{1}(\bar{I} ; H)$ wird nur im Existenzbeweis benutzt, sonst ist es hinreichend, $f \in C(\bar{I} ; H)$ anzunehmen.

\section{A-priori-Abschätzungen und die Eindeutigkeit der Lösung}

3.1. Wir erklären zuerst die Menge

$\mathcal{W}:=\left\{u \mid u \in C^{2}(\bar{I} ; H) \cap C^{1}(\bar{I} ; V) ; u(t) \in D(A), t \in \bar{I} ; A u \in C(\tilde{I} ; H)\right\}$.

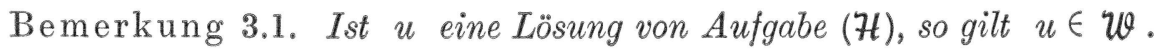


Satz 3.2. Für jedes $u \in W$ und für alle $t \in \bar{I}$ gelten die Abschätzungen

und

$$
\begin{gathered}
\left\|u^{\prime}(t)\right\|^{2}+\|u(t)\|_{V}^{2} \leqq c_{3} e^{c_{1} t}\left(\left\|u^{\prime}(0)\right\|^{2}+\|u(0)\|_{V}^{2}\right) \\
+c_{2} \int_{0}^{t} e^{c_{1}(t-s)}\left\|u^{\prime \prime}(s)+A u(s)+B u(s)\right\|^{2} d s
\end{gathered}
$$

$$
\begin{gathered}
\left\|u^{\prime}(t)\right\|^{2}+\|u(t)\|_{V}^{2} \geqq c_{5} e^{-c_{1} t}\left(\left\|u^{\prime}(0)\right\|^{2}+\|u(0)\|_{V}^{2}\right) \\
-c_{4} \int_{0}^{t} e^{c_{1}(s-t)}\left\|u^{\prime \prime}(s)+A u(s)+B u(s)\right\|^{2} d s
\end{gathered}
$$

mit $\quad c_{1}:=\max \left\{1+K_{b}, K_{b} k_{a}^{-1}\right\}, \quad c_{2}:=\max \left\{1, k_{a}^{-1}\right\}$, $c_{3}:=c_{2} \max \left\{1, K_{a}\right\}, c_{4}:=\min \left\{1, K_{a}^{-1}\right\}$ und $c_{5}:=c_{4} \min \left\{1, k_{a}\right\}$.

Beweis. Für die nach den Voraussetzungen stetig differenzierbare Funktion $\zeta$,

$$
\zeta(t):=\left\|u^{\prime}(t)\right\|^{2}+a(u(t)), \quad t \in \tilde{I},
$$

erhält man wegen (1.5) und (2.1)

$$
\zeta^{\prime}(t)=2 \operatorname{Re}\left(u^{\prime \prime}(t)+A u(t), u^{\prime}(t)\right),
$$

woraus nach (1.4) und (2.2) die Ungleichung

$$
\left|\zeta^{\prime}(t)\right| \leqq c_{1} \zeta(t)+\|f(t)\|^{2}
$$

mit $f(t):=u^{\prime \prime}(t)+A u(t)+B u(t)$ folgt. Hieraus ergibt sich einerseits

$$
\zeta(t) \leqq e^{c_{1} t} \zeta(0)+\int_{0}^{t} e^{c_{2}(t-s)}\|f(s)\|^{2} d s
$$

und andrerseits

$$
\zeta(t) \geqq e^{-c_{1} t} \zeta(0)-\int_{0}^{t} e^{c_{1}(s-t)}\|f(s)\|^{2} d s .
$$

Wegen (1.3) und (1.4) folgt nun Abschätzung (3.1) bzw. (3.2) aus (3.3) bzw. (3.4).

Folgerung 3.3. Für jedes $u \in W$ und für alle $t \in \bar{I}$ besteht die Abschätzung (die man der klassischen Energieungleichung zu entsprechen ansehen kann)

$$
\begin{aligned}
& \left\|u^{\prime}(t)\right\|^{2}+\|u(t)\|_{V}^{2} \\
& \leqq C\left(\left\|u^{\prime}(0)\right\|^{2}+\|u(0)\|_{V}^{2}+\|\| u^{\prime \prime}+A u+B u \|^{2}\right)
\end{aligned}
$$

mit einer Konstanten $C=C(T)>0$.

Auf Grund der Linearität von Aufgabe $(\mathcal{H})$ schließen wir nun das Resultat:

Folgerung 3.4. Die Lösung von Aufgabe (H) (wenn existiert) ist eindeutig. 


\section{Die Existenz der Lösung}

4.1. Für den Beweis betrachten wir den Hilbertraum $R:=L^{2}(I ; H) \times$ $V \times H$ versehen mit dem Skalarprodukt $(\cdot, \cdot)_{R}$,

$$
\left(r_{1}, r_{2}\right)_{R}:=\left(\left(f_{1}, f_{2}\right)\right)+\left(v_{1}, v_{2}\right)_{V}+\left(h_{1}, h_{2}\right),
$$

$r_{i}=\left(f_{i}, v_{i}, h_{i}\right) \in \mathcal{R}, \quad i=1,2$, und mit der zugehörigen Norm $\|\cdot\|_{R}$.

Durch die Definition

$$
\mathcal{D} w:=\left(w^{\prime \prime}+A w+B w, w(0), w^{\prime}(0)\right), \quad w \in \mathcal{W},
$$

wird eine lineare Abbildung $\mathcal{D}: \boldsymbol{W} \rightarrow R$ erklärt. Nun gilt

Lemma 4.1. Die Wertemenge $\mathcal{D}(\boldsymbol{W})$ ist dicht in $R$.

Beweis. A. Es sei $r=(f, v, h) \in R$ mit

$$
(r, \mathcal{D} w)_{R}=0, \quad w \in \mathcal{W},
$$

d. h. mit

$$
\left(\left(f, w^{\prime \prime}+A w+B w\right)\right)+(v, w(0))_{V}+\left(h, w^{\prime}(0)\right)=0, \quad w \in \mathfrak{W} .
$$

B. Wegen $f \in L^{2}(I ; H)$ gibt es nach [3], Corollar zu Satz 4.2, ein $f_{0} \in C(\vec{I} ; H)$ mit $D_{t} f_{0}=f$. Durch

$$
f_{1}(t):=-\int_{t}^{T} f_{0}(s) d s, \quad t \in \bar{I},
$$

und

$$
f_{2}(t):=\int_{t}^{T}(s-t) f_{0}(s) d s, \quad t \in \bar{I},
$$

erhält man die Funktionen $f_{i} \in C^{i}(\bar{I} ; H), \quad i=1,2$, mit

$$
f_{2}^{\prime \prime}=f_{1}^{\prime}=f_{0}, \quad f_{2}^{\prime}=f_{1} .
$$

Auf Grund von Satz 1.3 können wir

$$
u_{i}(t):=A^{-1} f_{i}(t), \quad t \in \bar{I}, \quad i=0,1,
$$

setzen. Nach Folgerung 1.4 und (4.5) schließt man dann

$$
u_{1} \in C^{1}(\bar{I} ; V), \quad u_{1}^{\prime}=u_{0} .
$$

C. Die stetig differenzierbare Funktion $\zeta$,

$$
\zeta(t):=\left\|f_{2}(t)\right\|^{2}+a\left(u_{1}(t)\right), \quad t \in \bar{I},
$$

erfüllt wegen (1.5), (2.1), (4.5), (4.6) und (4.7) die Beziehung

$$
\zeta^{\prime}(t)=2 \operatorname{Re}\left\{\left(f_{2}(t), f_{1}(t)\right)+\left(f_{0}(t), u_{1}(t)\right)\right\} .
$$


Nun konstruiert man eine Funktion $w \in W$ mit $w(0)=w^{\prime}(0)=0$ durch

$$
w(t):= \begin{cases}0, & 0 \leqq t \leqq t_{1}, \\ \frac{1}{4 !}\left(t-t_{1}\right)^{4} u_{1}\left(t_{2}\right), & t_{1} \leqq t \leqq T\end{cases}
$$

wobei $t_{1}, t_{2} \in \bar{I}$ beliebig gegeben sind. Für dieses $w$ findet man aus (4.3) nach [3], Corollar zu Satz 4.2, und gemäß (4.4) zuerst (man siehe [3])

$$
\left(f_{0}\left(t_{1}\right), u_{1}\left(t_{2}\right)\right)+\left(f_{2}\left(t_{1}\right), A u_{1}\left(t_{2}\right)+B u_{1}\left(t_{2}\right)\right)=0, \quad t_{1} \in \bar{I},
$$

und weiter mit $t=t_{1}=t_{2}$

$$
\left(f_{0}(t), u_{1}(t)\right)+\left(f_{2}(t), A u_{1}(t)+B u_{1}(t)\right)=0, \quad t \in \bar{I} .
$$

Mit Hilfe von (1.4), (2.2), (4.6) und der Ungleichung von Schwarz erhält man somit aus (4.8)

$$
-\zeta^{\prime}(t)=2 \operatorname{Re}\left(f_{2}(t), B u_{1}(t)\right) \leqq c \zeta(t), \quad t \in \bar{I},
$$

mit der Konstanten $c=K_{b} \max \left\{1, k_{a}^{-1}\right\}>0$. Da demzu $\zeta(T)=0$ ist, so gilt $\zeta(t) \leqq 0, \quad t \in \bar{I}$. Unmittelbar ist jedoch $\zeta(t) \geqq 0, \quad t \in \bar{I}$; deshalb besteht die Gleichung

$$
\zeta(t)=\left\|f_{2}(t)\right\|^{2}+a\left(u_{1}(t)\right)=0, \quad t \in \bar{I},
$$

woraus sich $f_{2}(t)=0, t \in \bar{I}$, und folglich $f=0$ ergibt.

D. Gleichung (4.3) nimmt hiernach die Gestalt

$$
(v, w(0))_{V}+\left(h, w^{\prime}(0)\right)=0, \quad w \in \mathcal{W} .
$$

Bei beliebigem $u \in D(A)$ gehört die durch $w(t):=u, \quad t \in \vec{I}$, erklärte Funktion $w$ zu w mit $w(0)=u, w^{\prime}(0)=0$. Also gilt

$$
(v, u)_{V}=0, \quad u \in D(A),
$$

und somit nach Folgerung 1.4 ist $v=0$. Deswegen hat man

$$
\left(h, w^{\prime}(0)\right)=0, \quad w \in W .
$$

Wählt man dabei als $w$ besonders die bei beliebigem $u \in D(A)$ durch $w(t):=t u, t \in \bar{I}$, definierte Funktion mit $w^{\prime}(0)=u$, so folgt

$$
(h, u)=0, \quad u \in D(A) \text {. }
$$

Hieraus schließt man $h=0$, wieder nach Folgerung 1.4 .

Bedingung (4.2) hat also $r=(f, v, h)=0$ zur Folge, d. h. $\bar{D}(w)$ ist dicht in $R$.

4.2. Mit Hilfe von Lemma 4.1 zeigen wir jetzt die Existenz einer Lösung für Aufgabe ( $\mathcal{H})$. 
4.2.1. Der Idee des Beweises liegen die Beziehungen

$$
\begin{aligned}
& v^{\prime \prime}(t)+A v(t)+B v(t)=f^{\prime}(t), \quad t \in \bar{I}, \\
& v(0)=g_{1}, \quad v^{\prime}(0)=f(0)-A g_{0}-B g_{0},
\end{aligned}
$$

zugrunde, die man durch formale Differentiation für $v=u^{\prime}$ aus den Gleichungen (2.3) und (2.4) herleitet.

Dabei gilt nun nach Voraussetzungen die Relation

$$
r:=\left(f^{\prime}, g_{1}, f(0)-A g_{0}-B g_{0}\right) \in R \text {; }
$$

deshalb gemäß Lemma 4.1 existiert eine Folge $\left\{w_{i}\right\}_{i=1}^{\infty} \subset \mathfrak{W}$ mit

d. h. mit

$$
\lim _{i \rightarrow \infty}\left\|r-\bar{D} w_{i}\right\|_{R}=0 \text {, }
$$

$$
\begin{aligned}
\lim _{i \rightarrow \infty}\left\{\left\|f^{\prime}-w_{i}^{\prime \prime}-A w_{i}-B w_{i}\right\|^{2}+\left\|g_{1}-w_{i}(0)\right\|_{V}^{2}\right. \\
\left.+\left\|f(0)-A g_{0}-B g_{0}-w_{i}^{\prime}(0)\right\|^{2}\right\}=0 .
\end{aligned}
$$

Nach (4.1) und Folgerung 3.3 kann man aus (4.9) die Existenz derartigen $w(t) \in V$ und $h(t) \in H$ für jedes $t \in \tilde{I}$ schließen, daß die Gleichungen

$$
\lim _{i \rightarrow \infty}\left\|w(t)-w_{i}(t)\right\|_{V}=0
$$

und

$$
\lim _{i \rightarrow \infty}\left\|h(t)-w_{i}^{\prime}(t)\right\|=0
$$

gleichmäßig in $\bar{I}$ erfüllt sind. Hieraus erhält man $w \in C(\bar{I} ; V)$ und $h \in C(\bar{I} ; H)$, sowie außerdem wegen $(4.10)$

$$
w(0)=g_{1} \text { und } h(0)=f(0)-A g_{0}-B g_{0} .
$$

Ferner findet man infolge der gleichmäßigen Konvergenz die Beziehung

$$
w(t)-w(0)=\int_{0}^{t} h(s) d s, \quad t \in \bar{I}
$$

daher ist $w \in C^{1}(\bar{I} ; H)$ und $w^{\prime}(t)=h(t), t \in \bar{I}$. Somit haben wir

$$
\begin{aligned}
& w \in C^{1}(\bar{I} ; H) \cap C(\bar{I} ; V), \\
& w(0)=g_{1}, \\
& w^{\prime}(0)=f(0)-A g_{0}-B g_{0} .
\end{aligned}
$$

4.2.2. Aus (4.10) ergibt sich

$$
\lim _{i \rightarrow \infty}\left\|\left|f^{\prime}-h_{i}\right|\right\|=0
$$


mit $h_{i}:=w_{i}^{\prime \prime}+A w_{i}+B w_{i}, \quad i=1,2, \ldots$ Nach (1.5) erhalten wir für alle $v \in V$

und folglich

$$
\begin{aligned}
a\left(w_{i}(t), v\right) & =\left(A w_{i}(t), v\right) \\
& =\left(h_{i}(t)-B w_{i}(t)-w_{i}^{\prime \prime}(t), v\right), \quad t \in \tilde{I},
\end{aligned}
$$

$$
\begin{aligned}
& \int_{0}^{t} a\left(w_{i}(s), v\right) d s \\
& =\left(\int_{0}^{t} h_{i}(s) d s-\int_{0}^{t} B w_{i}(s) d s-w_{i}^{\prime}(t)+w_{i}^{\prime}(0), v\right), \quad t \in \bar{I} .
\end{aligned}
$$

Hierbei haben wir auf Grund von (1.3) und (4.11) zuerst

$$
a\left(\int_{0}^{t} w(s) d s, v\right)=\lim _{i \rightarrow \infty} \int_{0}^{t} a\left(w_{i}(s), v\right) d s, \quad t \in \bar{I} .
$$

Aus (4.16) findet man

$$
\lim _{i \rightarrow \infty}\left(\int_{0}^{t} h_{i}(s) d s, v\right)=(f(t)-f(0), v), \quad t \in \tilde{I} .
$$

Weiter hat man wegen (2.2) und (4.11)

$$
\lim _{i \rightarrow \infty}\left(\int_{0}^{t} B w_{i}(s) d s, v\right)=\left(B \int_{0}^{t} w(s) d s, v\right), \quad t \in \tilde{I} .
$$

Somit erhalten wir aus (4.12), (4.17) bis (4.20) für alle $v \in V$ die Beziehung

$$
\begin{aligned}
& a\left(\int_{0}^{t} w(s) d s, v\right) \\
& =\left(f(t)-f(0)-B \int_{0}^{t} w(s) d s-w^{\prime}(t)+w^{\prime}(0), v\right), \quad t \in \bar{I} .
\end{aligned}
$$

4.2.3. Nun haben wir in Gleichung (4.21)

und

$$
\int_{0}^{t} w(s) d s \in V
$$

$$
f(t)-f(0)-B \int_{0}^{t} w(s) d s-w^{\prime}(t)+w^{\prime}(0) \in H .
$$

Nach 1.4 gelten also für jedes $t \in \tilde{I}$ die Relationen

$$
\int_{0}^{t} w(s) d s \in D(A)
$$

und 


$$
A \int_{0}^{t} w(s) d s=f(t)-f(0)-B \int_{0}^{t} w(s) d s-w^{\prime}(t)+w^{\prime}(0) .
$$

Wenn wir nun

$$
u(t):=g_{0}+\int_{0}^{t} w(s) d s, \quad t \in \bar{L},
$$

setzen, so haben wir $u(t) \in D(A), t \in \bar{I}$, und wegen (4.13) gilt

$$
u \in C^{2}(\bar{I} ; H) \cap C^{1}(\bar{I} ; V) .
$$

Für dieses $u$ besteht nach (4.15) und (4.22) die Gleichung

$$
u^{\prime \prime}(t)+A u(t)+B u(t)=f(t), \quad t \in \bar{I} .
$$

Da wir ferner $u(0)=g_{0}$ und wegen (4.14) auch $u^{\prime}(0)=g_{1}$ haben, so ist $u$ eine Lösung für Aufgabe ( $\mathcal{H})$.

\section{Aufgabe (H) ist korrekt gestellt}

5.1. Der Begriff „korrekt gestellt“ (im Sinne von Hadamard) für eine abstrakte Aufgabe mit gegebenen Anfangsdaten läßt sich in den folgenden Forderungen darstellen (man siehe etwa F. John [4], S. 273-275, M. M. Lavrentiev [6], S. 1-2, H. M. Lieberstein [7], S. 73-75):

1) die Aufgabe besitzt eine Lösung,

2) die Lösung ist eindeutig,

3) die Lösung hängt „stetig" von den Anfangsdaten ab.

Nach F. John haben die Anfangsdaten in der Aufgabe die Rolle der unabhängigen Ursachen, und die Lösung die der Wirkungen. Die Forderung nach der stetigen Abhängigkeit der Lösung bedeutet die Stabilität der Wirkungen, d. h. ,kleine“ Änderungen in den Anfangsdaten haben „kleine“ Änderung in der Lösung zur Folge.

5.2. Nun konkretisieren wir diesen Begriff für Aufgabe ( $\mathcal{H})$, d. h. wir stellen die „Stetigkeit“ in Bedingung 3) fest.

Die Änderung der Lösung $u$ und die Stabilität der Wirkungen lassen sich passend mit den Maximumnormen $\sup \left\{\|u(t)\|_{V} \mid t \in \bar{I}\right\}$ und $\sup \left\{\left\|u^{\prime}(t)\right\| \mid t \in \bar{I}\right\}$ abschätzen. Die Änderungen der Anfangsbedingungsfunktionen $g_{0}$ und $g_{1}$ kann man mit der $\|\cdot\|_{V}$-Norm und die des Nichthomogenitätsgliedes $f$ mit der Maximumnorm $\sup \{\|f(t)\| \mid t \in \bar{I}\}$ messen.

Wir haben jetzt das Resultat: 
Satz 5.1. Aufgabe () ist im obigen Sinne korrekt gestellt, d. h.

1) es existiert eine Lösung,

2) die Lösung ist eindeutig,

3) die Lösung u genügt der Bedingung:

Für jedes $\varepsilon>0$ gibt es Zahlen $\delta_{0}(\varepsilon)>0, \delta_{1}(\varepsilon)>0$ und $\delta(\varepsilon)>0$ derart, daß die Relationen

für

$$
\sup _{t \in \bar{I}}\|u(t)\|_{V} \leqq \varepsilon \quad \text { und } \sup _{t \in \bar{I}}\left\|u^{\prime}(t)\right\| \leqq \varepsilon
$$

$$
\left\|g_{0}\right\|_{V} \leqq \delta_{0}(\varepsilon), \quad\left\|g_{1}\right\|_{V} \leqq \delta_{1}(\varepsilon) \text { und } \sup _{t \in \bar{I}}\|f(t)\| \leqq \delta(\varepsilon)
$$

gelten.

Beweis. Auf Grund von Folgerung 3.4 und Abschnitt 4.2 sind die Forderungen 1) und 2) erfüllt, und auch 3) gilt. In der Tat, für die Lösung $u$ folgt aus (3.1) wegen Bemerkung 3.1 die Abschätzung

$$
\begin{aligned}
\sup _{t \in \bar{I}}\|u(t)\|_{V}^{2} & +\sup _{t \in \bar{I}}\left\|u^{\prime}(t)\right\|^{2} \\
& \leqq C\left\{\left\|g_{0}\right\|_{V}^{2}+\left\|g_{1}\right\|_{V}^{2}+\sup _{t \in \bar{I}}\|f(t)\|^{2}\right\},
\end{aligned}
$$

woraus die Behauptung sich ergibt.

5.3. Zuletzt bemerken wir noch, daß die in Satz 3.2 erreichten Abschätzungen (3.1) und (3.2) ziemlich schwach sind und daß mit deren Hilfe nichts Genaues über das Verhalten der Lösung zu sagen ist. Nämlich selbst im homogenen Fall lassen die Abschätzungen, nun von den Gestalten

$$
\left\|u^{\prime}(t)\right\|^{2}+\|u(t)\|_{V}^{2} \leqq c_{3} e^{c_{1} t}\left(\left\|u^{\prime}(0)\right\|^{2}+\|u(0)\|_{V}^{2}\right)
$$

und

$$
\left\|u^{\prime}(t)\right\|^{2}+\|u(t)\|_{V}^{2} \geqq c_{4} e^{-c_{1} t}\left(\left\|u^{\prime}(0)\right\|^{2}+\|u(0)\|_{V}^{2}\right),
$$

„zu viel Raum offen“ für die Lösung.

\section{Eine Anwendung auf eine hyperbolische Cauchysche Aufgabe mit nichthomogenen Randbedingungen}

6.0. Wir wenden jetzt die vorigen Ergebnisse auf die Theorie der linearen partiellen Differentialgleichungen an. Dafür führen wir zuerst einige Bezeichnungen ein. 
Es sei $\Omega$ eine offene nichtleere Menge in $R^{n}$. Wir setzen wie üblich

$$
\begin{aligned}
& C(\Omega)=C^{0}(\Omega):=\{u \mid u: \Omega \rightarrow C \text { stetig }\}, \\
& C_{b}(\Omega)=C_{b}^{0}(\Omega):=\{u \in C(\Omega) \mid u \text { beschränkt }\}, \\
& C_{0}(\Omega)=C_{0}^{0}(\Omega):=\{u \in C(\Omega) \mid \text { Supp } u \text { kompakt und in } \Omega\}, \\
& C^{m}(\Omega):=\left\{u \mid D^{p} u \in C(\Omega) \text { für alle } p \in N^{n},|p| \leqq m\right\}, \\
& C_{b}^{m}(\Omega):=\left\{u \mid D^{p} u \in C_{b}(\Omega) \text { für alle } p \in N^{n}, \quad|p| \leqq m\right\},
\end{aligned}
$$

wobei $m \in N_{+}$ist und $|p|:=p_{1}+\ldots+p_{n}$ für den Multiindex $p=$ $\left(p_{1}, \ldots, p_{n}\right) \in N^{n}$ und $D^{p}:=\left(\partial / \partial x_{1}\right)^{p_{1}} \ldots\left(\partial / \partial x_{n}\right)^{p_{n}}$ für den Differentialoperator $D=\left(\partial / \partial x_{1}, \ldots, \partial / \partial x_{n}\right)$ gesetzt wurden. Weiter sei $C^{\infty}(\Omega):=$ $\cap_{m=0}^{\infty} C^{m}(\Omega)$ und $C_{0}^{\infty}(\Omega):=C^{\infty}(\Omega) \cap C_{0}(\Omega)$.

In $C_{0}(\Omega)$ erklärt man das Skalarprodukt $(\cdot, \cdot)_{c}$ durch das Riemannsche Integral

$$
(u, v)_{c}:=\int u \bar{v} d x, \quad u, v \in C_{0}(\Omega)
$$

und schreibt $\|\cdot\|_{0}$ für die zugehörige Norm. Der Lebesgueraum $L^{2}(\Omega)$ wird nun durch $L^{2}(\Omega):=\mathcal{L}\left(C_{0}^{\infty}(\Omega) ; C\right)$ definiert, wobei $C_{0}^{\infty}(\Omega)$ mit dem Skalarprodukt $(\cdot, \cdot)_{c}$ versehen ist. Durch eine isometrische Einbettung liegt $C_{0}^{\infty}(\Omega)$ dann dicht im Raum $L^{2}(\Omega)$, der sich als ein Hilbertraum bezüglich des Skalarprodukts $(\cdot, \cdot)_{0}$ und der Norm $\|\cdot\|_{0}$ erweist, die die Erweiterungen von $(\cdot, \cdot)_{c}$ und $\|\cdot\|_{c}$ auf $L^{2}(\Omega)$ sind. Durch die Identifizierung ist weiter $C_{0}(\Omega) \subset L^{2}(\Omega)$, und es gilt $(u, v)_{0}=(u, v)_{c}$ für $u, v \in C_{0}(\Omega)$.

Die Menge $C_{0}^{\infty}(\Omega)$ versehen mit der Topologie von L. Schwartz ist der Testfunktionenraum $\mathcal{D}(\Omega)$, dessen topologischer Dualraum der Distributionenraum $\mathcal{D}^{\prime}(\Omega)$ ist. Nun hat man $L^{2}(\Omega) \subset \mathcal{D}^{\prime}(\Omega)$ und kann also für $u \in L^{2}(\Omega)$ und für jedes $p \in N^{n}$ die Ableitung $D^{p} u$ durch

$$
\begin{aligned}
\left\langle D^{p} u, \varphi\right\rangle & :=(-1)^{|p|}\left\langle u, D^{p} \varphi\right\rangle \\
& =(-1)^{|p|}\left(u, D^{p} \bar{\varphi}\right)_{0}, \quad \varphi \in \mathcal{D}(\Omega),
\end{aligned}
$$

erklären, wobei $\langle\cdot, \cdot\rangle$ das bilineare Dualprodukt zwischen $D(\Omega)$ und $D^{\prime}(\Omega)$ bezeichnet.

Für $m \in N$ bildet die Menge derjenigen $u \in L^{2}(\Omega)$, für die $D^{p} u \in L^{2}(\Omega)$ für alle $p \in N^{n},|p| \leqq m$, gilt, den Sobolevraum $H^{m}(\Omega)$. Er ist ein Hilbertraum bezüglich des Skalarprodukts $(\cdot, \cdot)_{m}$,

$$
(u, v)_{m}:=\sum_{|p| \leqq m}\left(D^{p} u, D^{p} v\right)_{0}, \quad u, v \in H^{m}(\Omega),
$$

und der zugehörigen Norm $\|\cdot\|_{m}$. Die Abschließung von $C_{0}^{\infty}(\Omega)$ in $H^{m}(\Omega)$ ist der mit $H_{0}^{m}(\Omega)$ bezeichnete abgeschlossene Teilraum von $H^{m}(\Omega)$. Besonders gilt $H_{0}^{0}(\Omega)=H^{0}(\Omega)=L^{2}(\Omega)$. 
6.1. Es sei $\mathcal{A}$ ein formaler (in der Variationsform gegebener) Differentialoperator in $\Omega$,

$$
\mathcal{A}:=\sum_{|p|,|q| \leqq m}(-1)^{|p|} D^{p} a_{p q}(x) D^{q},
$$

der Ordnung $2 m, m \in N_{+}$, und mit den Koeffizienten $a_{p q} \in C_{b}(\Omega)$. Dem Operator $\mathcal{A}$ fügen wir eine (Dirichletsche) sesquilineare Form

$$
a(\cdot, \cdot): H^{m}(\Omega) \times H^{m}(\Omega) \rightarrow C
$$

durch

$$
a(u, v):=\sum_{|p|,|q| \leqq m}\left(a_{p q} D^{q} u, D^{p} v\right)_{0}, \quad u, v \in H^{m}(\Omega),
$$

hinzu. Mit Hilfe von [9], S. 39, Lemma 5.1, und der Ungleichung von Schwarz erhält man

Lemma 6.1. Die Form $a(\cdot, \cdot)$ ist stetig in $H^{m}(\Omega) \times H^{m}(\Omega)$.

6.2. Für die Anwendung nehmen wir nun als $H$ den $\operatorname{Raum} H^{0}(\Omega)=$ $L^{2}(\Omega)$, und $V$ sei ein abgeschlossener Teilraum $H_{b \bar{d}}^{m}(\Omega)$ von $H^{m}(\Omega)$, $H_{0}^{m}(\Omega) \subset H_{b d}^{m}(\Omega) \subset H^{m}(\Omega)$, versehen mit dem Skalarprodukt $(\cdot, \cdot)_{m}$ und mit der Norm $\|\cdot\|_{m}$. Es sei bemerkt, daß $L^{2}(\Omega)$ und $H_{b d}^{m}(\Omega)$ wirklich ein Raumpaar (im Sinne von 1.1) bilden; denn Bedingung (1.1) ist unmittelbar erfüllt, und Bedingung (1.2) folgt daraus, da $\beta C_{0}^{\infty}(\Omega)$ dicht in $L^{2}(\Omega)$ ist (man siehe [9], S. 24).

Jetzt haben wir

Lemma 6.2. Es sei $u \in H^{m}(\Omega)$. Dann gilt die Relation

$$
a(u, \varphi)=\langle\mathcal{A} u, \bar{\varphi}\rangle, \quad \varphi \in \mathcal{D}(\Omega) .
$$

Im folgenden wird vorausgesetzt, daß die Form $a(\cdot, \cdot) \quad H_{b d}^{m}(\Omega)$-koerzitiv ist, d. h. es gibt eine Konstante $k_{a}>0$ mit

$$
|a(u)| \geqq k_{a}\|u\|_{m}^{2}, \quad u \in H_{b d}^{m}(\Omega) .
$$

Es bezeichne $A$ den von der Form $a(\cdot, \cdot)$ definierten (unbeschränkten) Operator bezüglich des Raumpaars $L^{2}(\Omega), H_{b d}^{m}(\Omega)$; dann gilt

$$
a(u, v)=(A u, v)_{0}, \quad u \in D(A), \quad v \in H_{b d}^{m}(\Omega) .
$$

Den Operator $A$ charakterisiert jetzt

Lemma 6.3. Es sei $D\left(A_{0}\right)$ die Menge derjenigen $u \in H_{b d}^{m}(\Omega)$, die den Bedingungen $\mathcal{A} u \in L^{2}(\Omega)$ und

$$
a(u, v)=(\mathcal{A} u, v)_{0}, \quad v \in H_{b d}^{m}(\Omega),
$$

genügen. Für den Operator $A_{0}:=\left.\mathcal{A}\right|_{D\left(A_{0}\right)}$ gilt dann $A_{0}=A$. 
Beweis. A. Es sei $u \in D\left(A_{0}\right)$. Dann ist für alle $v \in H_{b d}^{m}(\Omega)$

$$
|a(u, v)|=\left|(\mathcal{A} u, v)_{0}\right| \leqq\|\mathcal{A} u\|_{0}\|v\|_{0} .
$$

Somit ist die antilineare Abbildung

$$
v \mapsto a(u, v): H_{b d}^{m}(\Omega) \rightarrow C
$$

stetig für die $L^{2}(\Omega)$-Topologie, also gilt $u \in D(A)$.

B. Ist $u \in D(A)$, so hat man nach (6.1)

und besonders

$$
a(u, v)=(A u, v)_{0}, \quad v \in H_{b d}^{m}(\Omega),
$$

$$
a(u, \varphi)=(A u, \varphi)_{0}, \quad \varphi \in C_{0}^{\infty}(\Omega) .
$$

Nach Lemma 6.2 gilt somit

$$
\langle\mathcal{A} u, \varphi\rangle=a(u, \bar{\varphi})=(A u, \bar{\varphi})_{0}=\langle A u, \varphi\rangle
$$

für alle $\varphi \in \mathcal{D}(\Omega)$, d. h. $A u=\mathcal{A} u$ in $\mathcal{D}^{\prime}(\Omega)$. Deswegen erhalten wir $\mathcal{A} u=A u \in L^{2}(\Omega)$ und weiter

$$
(\mathcal{A} u, v)_{0}=(A u, v)_{0}=a(u, v), \quad v \in H_{b d}^{m}(\Omega) .
$$

Also gilt $D(A) \subset D\left(A_{0}\right)$ und $A_{0}=A$ in $D(A)$, woraus wegen Teil A die Gleichheit $A_{0}=A$ folgt.

Es sei $\mathcal{B}$ ein zweiter formaler partieller Differentialoperator der Ordnung $m_{0} \leqq m$ in $\Omega$,

$$
\mathcal{B}:=\sum_{|p| \leqq m_{0}} b_{p}(x) D^{p}
$$

mit den Koeffizienten $b_{p} \in C_{b}(\Omega)$. Dann ist der Operator $B:=\left.\mathfrak{B}\right|_{H_{b d}^{m}} ^{m}(\Omega)$ aus $\mathcal{L}\left(H_{b d}^{m}(\Omega) ; L^{2}(\Omega)\right)$.

Unsere bisherigen Annahmen sind also

und

$$
a_{p q} \in C_{b}(\Omega), \quad|p|,|q| \leqq m, \quad b_{p} \in C_{b}(\Omega), \quad|p| \leqq m_{0},
$$

$$
|a(u)| \geqq k_{a}\|u\|_{m}^{2}, \quad u \in H_{b d}^{m}(\Omega) ;
$$

überdies setze man voraus, daß

gilt.

$$
a(u, v)=\overline{a(v, u)}, \quad u, v \in H_{b d}^{m}(\Omega),
$$

6.3. Wenn wir jetzt Aufgabe ( $\mathcal{H})$ auf den obigen konkreten Fall anwenden, so erhalten wir die folgende hyperbolische Cauchysche Aufgabe mit nichthomogenen Randbedingungen:

Aufgabe $(\mathcal{H} b d) . \quad$ Es seien $g_{0} \in D(A) \quad\left(\subset H_{b d}^{m}(\Omega)\right), \quad g_{1} \in H_{b d}^{m}(\Omega)$ und $f \in C^{1}\left(\bar{I} ; L^{2}(\Omega)\right)$ gegeben. Gesucht ist eine Funktion $u$ mit den Eigenschaften: 
1) $u \in C^{2}\left(\vec{I} ; L^{2}(\Omega)\right) \cap C^{1}\left(\tilde{I} ; H_{b d}^{m}(\Omega)\right)$,

2) $u(t) \in D(A), \quad t \in \bar{I}$,

3) $u^{\prime \prime}(t)+A u(t)+B u(t)=f(t), \quad t \in \bar{I}$,

4) $u(0)=g_{0}, u^{\prime}(0)=g_{1}$.

Aus Satz 5.1 ergibt sich nun das folgende Resultat:

Satz 6.4. Aufgabe ( $\mathcal{H}$ bd) ist korrekt gestellt in dem in Satz 5.1 definierten Sinne.

Bemerkung. In Aufgabe ( $\mathcal{H} b d)$ sind die Randbedingungen ,frei“; deshalb kommen sie als Anfangsbedingungen implizit mit.

6.4. Zum Schluß zeigen wir, daß die z. B. in [3] untersuchte Situation als ein Spezialfall von Aufgabe $(\mathcal{H} b d)$ behandelt werden kann.

6.4.1. Mit unseren Bezeichnungen setzen wir also voraus, daß der Operator $\mathcal{A}$ stark elliptisch und formal selbstadjungiert in $\Omega$ ist, d. h. die Beziehungen

$$
\operatorname{Re} \sum_{|p|,|q|=m} \xi^{p} a_{p q}(x) \xi^{q} \geqq c_{1}|\xi|^{2 m}, \quad \xi \in \boldsymbol{R}^{n}, x \in \Omega,
$$

mit einer Konstanten $c_{1}>0$, wobei $\xi^{p}:=\xi_{1}^{p_{1}} \ldots \xi_{n}^{p_{n}}$ für $\xi=$ $\left(\xi_{1}, \ldots, \xi_{n}\right) \in \boldsymbol{R}^{n}$ und für $p=\left(p_{1}, \ldots, p_{n}\right) \in N^{n}$ gesetzt ist, und

$$
a_{p q}=\overline{a_{q p}}, \quad|p|,|q| \leqq m,
$$

bestehen, und daß ferner

$$
a_{p q} \in C_{b}^{|p|}(\Omega), \quad|p|,|q| \leqq m,
$$

gilt.

Als den Raum $H_{b d}^{m}(\Omega)$ nehmen wir nun den Raum $H_{0}^{m}(\Omega)$ der Dirichletschen Randbedingungen, und der Operator $B$ sei wie in 6.2 gegeben.

6.4.2. Mit Hilfe von (6.2), (6.4) und der Gårdingschen Ungleichung können wir stets annehmen (man siehe [3]), die Form $a(\cdot, \cdot)$ genüge der Abschätzung

$$
\operatorname{Re} a(u) \geqq c_{2}\|u\|_{m}^{2}, \quad u \in H_{0}^{m}(\Omega),
$$

folglich wegen (6.3)

$$
a(u) \geqq c_{2}\|u\|_{m}^{2}, \quad u \in H_{0}^{m}(\Omega),
$$

mit einer Konstanten $c_{2}>0$. Aus (6.3) folgt auch (man beachte [9], S. 40)

$$
a(u, v)=\overline{a(v, u)}, \quad u, v \in H_{0}^{m}(\Omega) .
$$

Für den von $a(\cdot, \cdot)$ definierten (unbeschränkten) Operator $A$ bezüglich des Raumpaars $L^{2}(\Omega), H_{0}^{m}(\Omega)$ besteht jetzt die Beziehung 


$$
D(A)=\left\{u \in H_{0}^{m}(\Omega) \mid \mathcal{A} u \in L^{2}(\Omega)\right\} .
$$

In der Tat, ist $u \in H_{0}^{m}(\Omega)$ mit $\mathcal{A} u \in L^{2}(\Omega)$, so haben wir nach Lemma 6.2

$$
a(u, \varphi)=\langle\mathcal{A} u, \bar{\varphi}\rangle=(\mathcal{A} u, \varphi)_{0}, \quad \varphi \in \mathcal{D}(\Omega) .
$$

Somit wegen Lemma 6.1 gilt auch

$$
a(u, v)=(\mathcal{A} u, v)_{0}, \quad v \in H_{0}^{m}(\Omega),
$$

weil $C_{0}^{\infty}(\Omega)$ dicht in $H_{0}^{m}(\Omega)$ liegt. Hieraus und aus Lemma 6.3 folgt (6.5).

Bemerkung. a) In den vorigen Überlegungen ist tatsächlich hinreichend, statt (6.4) die Relationen $a_{p q} \in C_{b}(\Omega), \quad|p|,|q| \leqq m$, anzunehmen, und überdies für die mögliche Anwendung der Gårdingschen Ungleichung, daß die Koeffizienten $a_{p q}, \quad|p|=|q|=m$, gleichmäßig stetig in $\Omega$ sind.

b) Unter Voraussetzung (6.4) hat man $C_{0}^{\infty}(\Omega) \subset D(A)$, woraus sich ergibt, daß $D(A)$ dicht sowohl in $L^{2}(\Omega)$ als auch in $H_{0}^{m}(\Omega)$ ist. Diese Tatsache wissen wir jedoch schon auf Grund von Folgerung 1.4.

6.4.3. In der in 6.4.1 und 6.4.2 vorkommenden Situation wird Aufgabe $(\mathcal{H} b d)$ auf eine der z. B. in [3] betrachteten Aufgabe äquivalente Aufgabe reduziert, die Aufgabe ( $\mathcal{H} 0$ ) heiße.

Besonders folgt die in [3], Sätze 6.2 und 7.1, bewiesene Existenz einer eindeutigen Lösung für Aufgabe ( $\mathcal{H}$ 0) jetzt aus Satz 6.4.

\section{Literatur}

[1] Dremmann, J.: Über das Dirichletsche Rand- und Anfangswertproblem für die Wellengleichung. - Methoden und Verfahren der Mathematischen Physik 4, B.I-Hochschulskripten 723a/b, 33-44, Bibliographisches Institut, Mannheim, 1971.

[2] Dremmann, J., und P. Werner: Über das Dirichletsche Rand- und Anfangswertproblem für hyperbolische Differentialgleichungen. - Applicable Anal. $2,1972,131-141$.

[3] -»- Ein distributionentheoretischer Aufbau der Theorie der Bochner-Räume und Anwendungen auf das Dirichletsche Rand- und Anfangswertproblem für hyperbolische Differentialgleichungen. - Erscheint demnächst in Ann. Acad. Sci. Fenn. Ser. A I.

[4] Jонм, F.: Partial differential equations. - Mathematics applied to physics, $229-315$, UNESCO. Paris / Springer-Verlag, Berlin - Heidelberg - New York, 1970.

[5] Ladyženskaja, O. A.: On the solution of nonstationary operator equations. Amer. Math. Soc. Transl. (2) 65, 1967, 200-236.

[6] Lavrentiev, M. M.: Some improperly posed problems of mathematical physics. Springer tracts in natural philosophy 11, Springer-Verlag, Berlin - Heidelberg - New York, 1967. 
[7] Lieberstern, H. M.: Theory of partial differential equations. - Mathematics in science and engineering 93, Academic Press, New York - London, 1972.

[8] VIŠır, M. I., und O. A. LADYžEnskAyA : Boundary value problems for partial differential equations and certain classes of operator equations. - Amer. Math. Soc. Transl. (2) 10, 1958, 223-281.

[9] Werner, P.: A distribution-theoretical approach to certain Lebesgue and Sobolev spaces. - J. Math. Anal. Appl. 29, 1970, 18-78.

[10] Wilcox, C. H.: Initial-boundary value problems for linear hyperbolic partial differential equations of the second order. - Arch. Rational Mech. Anal. 10, $1962,361-400$.

\section{Universität Jyväskylä \\ Mathematisches Institut \\ Sammonkatu 6 \\ SF-40 100 Jyväskylä 10 \\ Finnland}

Eingegangen am 26. November 1974 